\title{
Candida prevalence and oral hygiene due to orthodontic therapy with conventional brackets
}

\author{
Kinga Grzegocka ${ }^{1 *} \mathbb{D}$, Paweł Krzyściak² ${ }^{2}$, Anna Hille-Padalis ${ }^{1} \mathbb{D}$, Jolanta E. Loster ${ }^{3} \mathbb{D}$,
} Katarzyna Talaga-Ćwiertnia² (B) and Bartłomiej W. Loster ${ }^{1}$ (D)

\begin{abstract}
Background: Conventional brackets are often used during orthodontic therapy of patients with malocclusion. The complex construction of such brackets greatly inhibits oral hygiene, which predisposes to increased carriage of microbiota. Orthodontic brackets could act as reservoir of yeast and predispose to oral candidosis. The aim of this study was to assess Candida prevalence and the role of oral hygiene during fixed appliance therapy. A further aim was to characterize the isolated yeasts according to their ability to form biofilms.

Methods: Seventeen participants (average age $17 \pm 7$ years) were monitored by taking oral rinses and elastomeric ligature samples, and by evaluating the approximal plaque index (API) and gingival bleeding index (GBI) before and after placement of the orthodontic conventional brackets for 12 weeks. Isolated yeasts were counted and biofilm formation was evaluated.

Results: One hundred and sixteen samples (67 oral rinses and 49 orthodontic elastomers) were collected. Ten patients (58.8\% subjects) were Candida-carriers (two were colonized after bracket placement) and C. albicans was the most common species. The average number of yeasts in the oral cavity showed some fluctuation during the study, but in general had an upward trend (adj. R2 $=0.7967, p=0.07025$ ). A correlation was found between median number of yeasts and the periodontal indices (API, GBI). The average API values decreased in the Candida-carriers (adj. $R^{2}=0.95 ; p=0.01709$ ), while average $\mathrm{GBI}$ values increased in the noncarriers (adj. $R^{2}=0.92 ; p=0.0256$ ).

Conclusions: Treatment with orthodontic appliances promotes Candida yeast colonization, which is variable over time in terms of strain and species, with dominance of $C$. albicans, and without increased biofilm-forming activity. The API value decreases over time in carriers, and the GBI value increases in uncolonized patients, which may have predictive significance for the development of oral candidiasis during orthodontic treatment.
\end{abstract}

Keywords: Orthodontic brackets, Candida, Oral hygiene, Periodontal index

\section{Background}

Conventional brackets are often used during orthodontic therapy of patients with malocclusion. The complex

\footnotetext{
* Correspondence: kinga.grzegocka@uj.edu.pl

${ }^{1}$ Department of Orthodontics, Dental Institute, Faculty of Medicine, Jagiellonian University Medical College, ul. Montelupich 4/108, 31-155 Kraków, Poland

Full list of author information is available at the end of the article
}

construction of such brackets greatly inhibits oral hygiene, which predisposes to increased carriage of bacteria and yeasts $[1,2]$. An increased amount of fungi in the oral cavity can, with predisposing factors, lead to the development of oral mycosis. A count of yeast colonies (colony-forming unit, CFU) can be used to estimate the number of fungi, calculated from the volume of washings collected from the patient's mouth. This method

(c) The Author(s). 2020 Open Access This article is licensed under a Creative Commons Attribution 4.0 International License, which permits use, sharing, adaptation, distribution and reproduction in any medium or format, as long as you give appropriate credit to the original author(s) and the source, provide a link to the Creative Commons licence, and indicate if changes were made. The images or other third party material in this article are included in the article's Creative Commons licence, unless indicated otherwise in a credit line to the material. If material is not included in the article's Creative Commons licence and your intended use is not permitted by statutory regulation or exceeds the permitted use, you will need to obtain permission directly from the copyright holder. To view a copy of this licence, visit http://creativecommons.org/licenses/by/4.0/ The Creative Commons Public Domain Dedication waiver (http://creativecommons.org/publicdomain/zero/1.0/) applies to the data made available in this article, unless otherwise stated in a credit line to the data. 
allows both the number composition (quantitative assessment) and species composition (qualitative assessment) of isolated colonies to be determined, unlike swabs, which are less reliable for technical reasons (they require sampling from a specific surface area (e.g., $1 \mathrm{~cm}^{2}$ ) or the same location (such as the palate; they also involve a high risk of contamination through touching other anatomical structures, and are prone to errors related to adherence and recovery of colonies from a swab) [3]. The state of oral hygiene can also be expressed numerically, as with the periodontological indices. One of the most popular dental indicators is the approximal plaque index (API), which expresses the amount of uncleaned interdental space as a percentage. API values of $25-39 \%$ indicate good hygiene, and $<25 \%$ point to optimal hygiene. A permanent lack of good oral hygiene causes inflammation of the gums, the first symptom of which is bleeding. The gingival bleeding index (GBI) is used to assess gum inflammation. GBI values of below $10 \%$ indicate clinically healthy periodontium.

One element of conventional orthodontic brackets are elastomeric rings, which are used to connect the bracket with the orthodontic wires. The irregularity and roughness of the surface of these rings are favorable to colonization and biofilm formation by microorganisms [4, 5]. Biofilms are usually multispecies assemblages of microorganisms encased in a matrix a mode of life common to most microorganisms in natural and medical systems (as well as components of orthodontic appliances), which allows survival in hostile environments. A biofilm can become a reservoir of pathogens and, with predisposing factors, can contribute to thrush and other forms of oropharyngeal candidosis. One factor in Candida's virulence is the ability of yeasts to form biofilms, and it would seem that strains isolated from patients colonized and using orthodontic appliances will form a biofilm well $[6,7]$.

Even though Candida is a part of the normal oral microbiota, being found in $17-75 \%$ human population, it can often be the cause of oral mycoses, especially in immunodeficient patients $[8,9]$. The most common etiological factor of oral candidosis is Candida albicans. However, C. tropicalis, C. glabrata, C. parapsilosis, C. krusei sometimes occur with high prevalence, especially in susceptible patients such as diabetics [10-12]. All these species have a great ability to form biofilms, especially with oral gram positive bacteria [8-11].

Very few studies have compared Candida prevalence and Candida growth in orthodontic patients before, during, and after treatment $[1,12]$. The small number of papers related to this topic and the correlation between orthodontic elastomeric rings and oral Candida growth led to this study.
The purpose of this study was to investigate the occurrence of Candida species and the role of oral hygiene, as measured by periodontal parameters during fixed appliance therapy. A further aim was to characterize the isolated Candida species by their ability to form biofilms, which are a virulence factor in the development of candidosis related to orthodontic therapy.

\section{Methods \\ Patients and samples}

Seventeen patients (eleven females and six males, aged 11-30 years old, average age $17 \pm 7$ years, median 14 years) at the Department of Orthodontics, University Dental Clinic, Kraków, Poland, were randomly selected. Due to occlusion defects, all subjects required orthodontic treatment using conventional brackets and gave their written consent to participate in the study. The inclusion criteria were healthy individuals, both sexes, aged $\leq 30$ years old. The exclusion criteria were oral mucosa disease; smoking; use of antibiotics, corticosteroids, or any hormone medication in the 3 months prior to the study; pregnancy, or breastfeeding. All patients were informed that the use of antimicrobial mouthwashes was prohibited during the study.

The research project included a schedule of four visits: T0: before bonding brackets; $\mathrm{T} 1, \mathrm{~T} 2$, and $\mathrm{T} 3$ : approximately 2, 6, and 12 weeks after bonding brackets, respectively.

During the study, patients used the same type elastic rings (color Glow Blue, catalogue index OCLGB, Orthodontic Design and Production, USA), metal brackets Cannon Ultra System (Orthodontic Design and Production, USA), and NiTi wires (Fairfield Orthodontics, USA).

All participants were thoroughly instructed by one of the authors on how to properly take care of oral hygiene during orthodontic treatment. Patients reported for all appointments properly prepared (in accordance with written recommendations): in the morning, on an empty stomach (minimum $6 \mathrm{~h}$ after eating), before morning toothbrushing and other oral hygiene procedures. Patients were also asked to limit exercise and follow a mixed diet on the day preceding the study.

During visit T0, before brackets were bonding, oral hygiene was assessed using the approximal plaque index (API), following Lange, and the gingival bleeding index (GBI), following Ainamo and Bay. Oral rinses were collected.

To measure API, a periodontal probe was gently guided through the approximal spaces of the first and third quadrants from the oral aspect, and of the second and fourth quadrants from the buccal aspect. The presence of plaque deposits was recorded as a positive result. 
The percentage of sites with positive results was counted.

The GBI was taken by gently probing the orifice of the gingival crevice. If bleeding occurred within $10 \mathrm{~s}$, a positive finding was recorded. The number of positive sites was recorded and then expressed as a percentage of the number of sites examined.

Collection of mycological material was performed using oral rinses. Patients under the supervision of the orthodontist rinsed their mouth for $60 \mathrm{~s}$ with $10 \mathrm{ml}$ isotonic saline $(0.9 \% \mathrm{NaCl})$ at room temperature, and spat out the rinses into a sterile container, which was immediately delivered to the mycological laboratory.

During visits T1, T2, and T3, the API and GBI indices were re-evaluated, and oral rinses and elastomeric rings were collected. Orthodontic ligatures were placed into Eppendorf tubes filled with saline solution. Elastomers were collected using a sterile dental kit in aseptic conditions to prevent material contamination.

\section{Microbiological analysis Total candida and mean candida carriage}

The mouth washing samples were vigorously shaken for 90 s with a Vortex shaker, and then quantitatively plated on Sabouraud's chloramphenicol agar (Biocorp) and incubated at $35 \pm 2{ }^{\circ} \mathrm{C}$ for $72 \mathrm{~h}$. The collected elastomeric ligatures were inoculated directly onto the medium. The grown colonies were identified based on classical mycological methods, such as colony morphology, the Dalmau plate technique, and an API 20C AUX commercial assimilation test (Biomerieux).

Strains were collected and frozen for further biofilm formation studies.

\section{In vivo biofilm scanning electron microscopy (SEM)}

Several randomly selected ligatures, present in the oral cavity for about 4 weeks, with known Candida growth were subjected to a scanning electron microscope analysis to assess the biofilm produced in vivo. The topography of an unused elastic ligature was also examined as a control. Analysis of the prepared samples was performed using a JEOL JSM-35CF scanning microscope (JSM-35CF; JEOL Vacuum Evaporator) at the Laboratory of the Otolaryngology Clinic, University Hospital, Kraków.

\section{Candida biofilm formation}

The biofilm formation assay was performed as follows: the overnight culture of investigated strains was transferred to sterile saline, and the fungal suspension was adjusted to 1 on McFarland scale with a densitometer (DEN1 Biosan, Lithuania). One hundred $\mu \mathrm{L}$ of standardized suspensions (eight wells per strain) were added to each well of sterile 96-well flat-bottom polystyrene plates filled previously $(100 \mu \mathrm{L}$ per well) with double concentrated Roswell Park Memorial Institute (RPMI) 1640 medium with L-glutamine without bicarbonate (Sigma Aldrich), supplemented with $2 \%$ glucose (Avantor Performance Materials, Gliwice, Poland) and buffered with 3-morpholinopropane-1-sulfonic acid (MOPS, Sigma Aldrich). The plates were incubated for $1.5 \mathrm{~h}$ at $37^{\circ} \mathrm{C}$ for the adherence phase, before being washed twice with sterile phosphate buffered saline (PBS) to remove nonadherent cells. Each well was then filled with new RPMI medium and incubated without shaking for $72 \mathrm{~h}$ at $37^{\circ} \mathrm{C}$. After that time, the plates were washed with PBS, dried in air for $45 \mathrm{~min}$, and stained with $125 \mu \mathrm{L}$ per well of $0.1 \%$ crystal violet solution (Avantor Performance Materials, Gliwice, Poland) for $45 \mathrm{~min}$ at room temperature. The microtiter dish was then washed and then dried. A $150 \mu \mathrm{L}$ volume of $95 \%$ ethanol (Avantor Performance Materials, Gliwice, Poland) was added to each well, and then plates were covered and incubated for $45 \mathrm{~min}$ at room temperature. A $100 \mu \mathrm{L}$ sample of the resulting ethanol-crystal violet solution was then transferred from each well to a new microtiter plate, and the optical density (OD) was determined at a wavelength of $570 \mathrm{~nm}$ (Infinite 200 Pro Tecan Männedorf, Switzerland). The experiment was repeated, with each strain being analyzed in sixteen replicates.

The study included 27 isolates from ten patients and one reference strain of $C$. albicans (ATCC 90028).

\section{Statistical analysis}

To detect differences in GBI, API, and number of Candida colony-forming unit (CFU) across multiple tests, we used one-way repeated measures analysis of variance by ranks (Friedman test and Skillings-Mack test). Differences in biofilm formation among Candida strains were evaluated using the Kruskal-Wallis test with Dunn's post hoc analysis. All statistical analysis was carried out using $\mathrm{R}$ software [13, 14], and a $p$-value below 0.05 was considered significant.

\section{Results \\ Candida carriage}

One hundred and sixteen samples were collected from seventeen patients (67 oral rinses and 49 orthodontic ligatures samples). Positive Candida growth was noted in 52 samples (34 oral rinses comprising 51\% of samples, and 18 orthodontic ligatures samples comprising 37\%) and $C$. albicans was the most commonly isolated species (91.1\%), followed by C. tropicalis (4.5\%) and C. guilliermondii (4.5\%).

Analysis of Candida carriage among oral rinses before bonding brackets shown that eight patients (47\%) had Candida with an average number of $5.5 \times 10^{2} \pm 4.8 \times 10^{2}$ $\mathrm{CFU} / \mathrm{ml}$. Two further patients $(11.8 \%)$ were colonized 
during the study. In total, ten patients $(58.8 \%)$ were Candida-carriers, of which 6 (35\%) were consistent carriers (positive results throughout the whole study) and four (23.5\%) were inconsistent carriers (negative result at least once in the study). The average number of yeast colonies fluctuated over the study, with little decline at stage T2. At stages T0, T1, T2, and T3, the Candidacarriers had $4.4 \times 10^{2}, 8.8 \times 10^{2}, 8 \times 10^{2}, 190 \times 10^{2} \mathrm{CFU} /$ $\mathrm{ml}$, respectively (Table 1 ). The highest CFU value of $8.5 \times 10^{3} \mathrm{CFU} / \mathrm{ml}$ was found in one patient (P.M.) in stage T3. The average number of colonies per unit volume obtained from oral rinses showed an upward trend depending on the study stage (adjusted $R^{2}=0.7967$ ), but without statistical significance $(p=0.07025)$ (Fig. 1).

\section{$\mathrm{API}$ and $\mathrm{GBI}$ results}

The mean API and GBI values at stage T0 were $41 \% \pm$ 22 and $10 \% \pm 9$, respectively. There were no differences in the distribution of GBI values for all patients between study stages (Friedman rank sum test chi-squared = 2.4041, $\mathrm{df}=3, p$-value $=0.4929)$ or differences in API values (Friedman rank sum test; chi-squared $=1.0185$, $\mathrm{df}=3, p$-value $=0.7968$ ).

When the study groups were divided into two subgroups: (1) non-Candida-carriers, comprising $41 \%$, and (2) Candida-carriers (patients in whom yeast growth was found at any stage of sampling), comprising 59\%, some tendencies for changes in median index values were observed. In Candida-carriers, medians of API values decreased (adjusted R-squared coefficient $=0.94$; $p=0.01709$ ), while in non-Candida-carriers, the medians of GBI values increased (adjusted R-squared coefficient $=0.92 ; p=0.0256$ ) (Fig. 2a, b).

\section{Biofilm formation: SEM results}

Topographic assessment of unused elastic rings under an electron microscope showed a clean surface, free of microorganisms (Fig. 3a).

On the surface of the colonized orthodontic elastic ligatures, microorganisms created a multicellular, architecturally complex structure, with the presence of various types of bacteria (cocci, bacilli, rods) and yeast (both early and late stage of biofilm formation) (Fig. 3b, c, d).

\section{Ability to form biofilm}

Most of the examined strains created biofilm (Fig. 4). Pairwise comparisons with control [14] shows the difference in biofilm production for seven strains (Dunn's post hoc test after Kruskal-Wallis test; $p<0.05$ ). These strains $(59,28,65,25,30,52,38)$ produced significantly more biofilm biomass than Candida albicans ATCC 90028.

In the overwhelming majority of cases, biofilm formation was homogenous among strains isolated from particular patients (F.A., F.P., O.U., P.J., P. M., R.A., and S.P.). In only few cases, when various species were isolated (i.e., patient D.M.2, and K.J.) and in one case of Candida albicans isolation (D.M.), the biofilm formation differed by study stage.

There is no correlation between biofilm forming ability and API or GBI index (adjusted $R^{2}=0.02, p=0.2377$, adj. $R^{2}=0.04, p=0.1824$, respectively).

\section{Discussion}

In the current study, the percentage of Candida-carriers before orthodontic treatment was relatively high, at $47 \%$ of patients. It increased during orthodontic treatment to almost $59 \%$ (nearly $12 \%$ of subjects became carriers). This would not be expected given the occurrence of Candida in the Polish population, affecting an average of $30.6 \%$ of healthy individuals [15]. However, the study citied used oral swabs $(N=654 ; 7-45$ years old $)$ and the lower result may be the effect of the different collecting method. Tooyama et al. [3] showed that the test result depends significantly on the sampling method. In his study of Japanese patients $(N=200$, average age 47.2 years old), when measured using oral swabs, Candida affected $33.5 \%$ of patients, whereas $52 \%$ of the same group of patients were found to be colonized with Candida sp. when concentrated rinse was used as the collecting method, comparable with our results and methodology. This shows that the method of sampling has a great

Table 1 Average value of Candida sp. (CFU/ml) and API and GBI indices (\%) during the study for all patients

\begin{tabular}{|c|c|c|c|c|c|c|}
\hline & & TO & $\mathrm{T1}$ & $\mathrm{T} 2$ & T3 & $\begin{array}{l}\text { Differences between } \\
\text { stages }\end{array}$ \\
\hline $\begin{array}{l}\text { Candida colonization } \\
{[\text { CFU/ml count] }}\end{array}$ & $\begin{array}{l}\text { Average } \pm \\
\text { SD (range) } \\
\text { Median }\end{array}$ & $\begin{array}{l}2.6 \times 10^{2} \pm 4.3 \times \\
10^{2}\left(0-1.3 \times 10^{3}\right) \\
0 \times 10^{2}\end{array}$ & $\begin{array}{l}5.2 \times 10^{2} \pm 9.4 \times \\
10^{2}\left(0-3.0 \times 10^{3}\right) \\
1 \times 10^{2}\end{array}$ & $\begin{array}{l}4.7 \times 10^{2} \pm 7.6 \times \\
10^{2}\left(0-3.0 \times 10^{3}\right) \\
2 \times 10^{2}\end{array}$ & $\begin{array}{l}10.7 \times 10^{2} \pm 23.1 \times \\
10^{2}\left(0-8.5 \times 10^{3}\right) \\
1 \times 10^{2}\end{array}$ & $\begin{array}{l}p=0.9092 \text { (Skillings-Mack } \\
\text { Statistic) }\end{array}$ \\
\hline API value [\%] & $\begin{array}{l}\text { Average } \pm \\
\text { SD (range) } \\
\text { Median }\end{array}$ & $\begin{array}{l}40.9 \pm 22(13-94.8) \\
42.3\end{array}$ & $\begin{array}{l}43.4 \pm 22.6(13.6- \\
88.2) \\
38.0\end{array}$ & $\begin{array}{l}38.3 \pm 17.4(10- \\
66.7) \\
35.3\end{array}$ & $\begin{array}{l}39.8 \pm 23(4.5-100) \\
35.0\end{array}$ & $\begin{array}{l}p=0.7968 \text { (Friedman rank } \\
\text { sum test; chi-squared) }\end{array}$ \\
\hline GBI value [\%] & $\begin{array}{l}\text { Average } \pm \\
\text { SD (range) } \\
\text { Median }\end{array}$ & $\begin{array}{l}10.3 \pm 9(0-25) \\
8.3\end{array}$ & $\begin{array}{l}9.4 \pm 8.8(0-31.2) \\
7.5\end{array}$ & $\begin{array}{l}19.23 \pm 24.8(0- \\
93.8) \\
11.8\end{array}$ & $\begin{array}{l}10.6 \pm 8.5(0-29.5) \\
12.5\end{array}$ & $\begin{array}{l}p=0.4929 \text { (Friedman rank } \\
\text { sum test; } \text { chi-squared) }\end{array}$ \\
\hline
\end{tabular}

Legend: $A P I$ approximal plaque index, GBI gingival bleeding index, $S D$ standard deviation, CFU colony-forming unit 


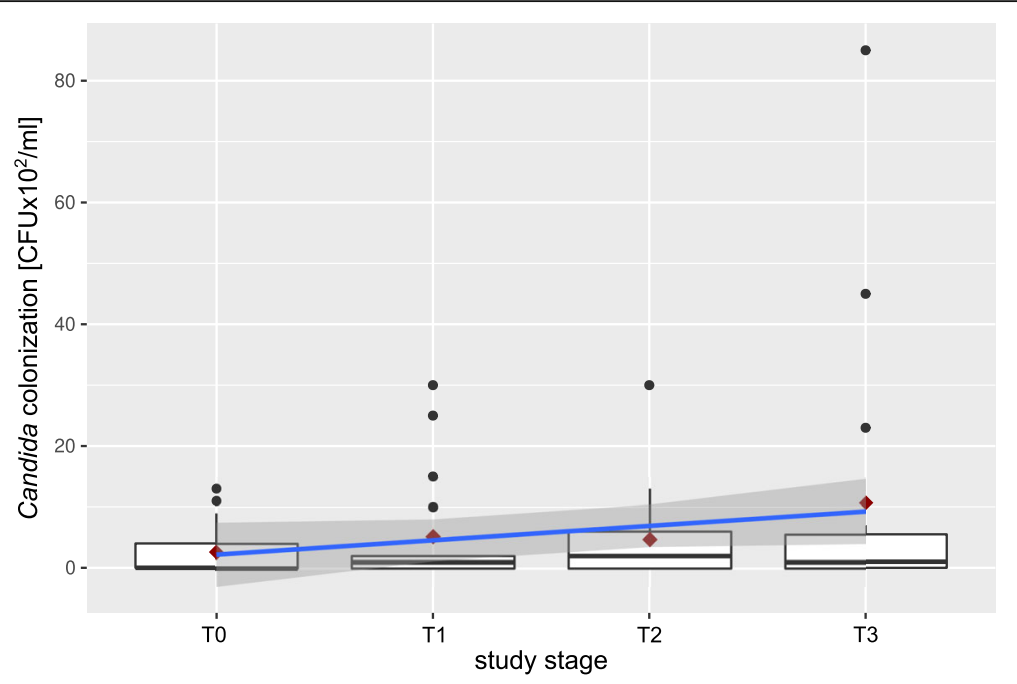

Fig. 1 Occurrence of Candida in the oral cavity of Candida carriers throughout the study (shown as a boxplot with average number of colonies). The blue line connecting the averages ( shows an upward trend; the grey area is the confidence interval for the mean

impact on the results, and that not all studies can be directly compared.

Zheng et al. [16] determined that oral Candida sp. was present in only $14 \%$ of young Chinese adults $(N=50$, average age 13.6 years old) before the application of fixed orthodontic appliance. This lower result may be the effect of toothbrushing prior to sampling. Arslan et al. [17] tested a Turkish population $(N=72$, average age 19.6 years old) using saliva samples and oral swabs, finding high Candida-carriage rate of $58.5 \%$. This may suggest the occurrence of large population variables, both within age and ethnic groups, as carriage of fungi depends on many factors, such as diet and lifestyle.

In our investigation, two patients (nearly 12\%) became Candida-carriers over the course of the experimental phase of the study (after bracket placement). This is coherent with other publications that have suggested that treatment with orthodontic appliances promotes Candida yeast colonization. Hägg et al. [18] demonstrated using the imprint technique that overall Candida prevalence increases after bonding brackets $(N=27$, mean age 15 years old).

Lee et al. [19] described that $15 \%$ of subjects became Candida-carriers in their study using oral concentrated rinse analysis of Chinese patients $(N=112$, average age 17.7 years old), amount to an increase in Candida-carriers from an initial 32\% (T0: before bracket bonding) to a maximum of $50 \%$ (T5: around 5 months after bonding). They reported that $11 \%$ of subjects were consistent carriers of Candida species, $64 \%$ were inconsistent carriers, and only $25 \%$ were consistent non-carriers (i.e., never carried Candida species throughout the experimental period of 12 months). We were also able to distinguish a group of consistent (35\%) and inconsistent
(23.5\%) Candida-carriers. This may indicate that the initially tested noncarrier group at the study baseline contained some false negative results. It needs to be considered that the introduction of orthodontic appliance is only one variable factor influencing the prevalence of Candida yeast in the oral cavity (others being diet, oral hygiene, lifestyle, immune system, etc.). In future studies, longer observation of the study group with multiple tests is recommended to isolate the group of consistent and inconsistent carriers before the start of the experimental phase of the study.

In our study, a quantitative analysis of Candida colonies in carriers group was carried out, as the number of yeast colonies indicates development of oral yeast infection during orthodontic treatment. Tooyama et al. [3] determined the reference ranges of Candida colonies for healthy commensal carriages at $0-5 \mathrm{CFU} / \mathrm{swab}$ and 0 $670 \mathrm{CFU} / \mathrm{ml}$ with the concentrated rinse method. In our study, five patients had an average colony count higher than $670 \mathrm{CFU} / \mathrm{ml}$, but clinically did not show any symptoms suggesting that Candida grew on the abiotic surface of dental appliances, rather than on the mucosa. Analysis of the quantitative data shows that the number of colonies differs by time and by patient. However, in our research, we found no statistically significant differences in the number of Candida sp. during the 12 weeks of study. Despite this, our results suggest a positive correlation between the average number of colonies and the duration of study. The amount of oral Candida may fluctuate, and the lack of statistical significance may be due to the hygiene habits of patients, the food they consume, yeast biology, the small research sample, or high initial number of oral Candida-carriers. 


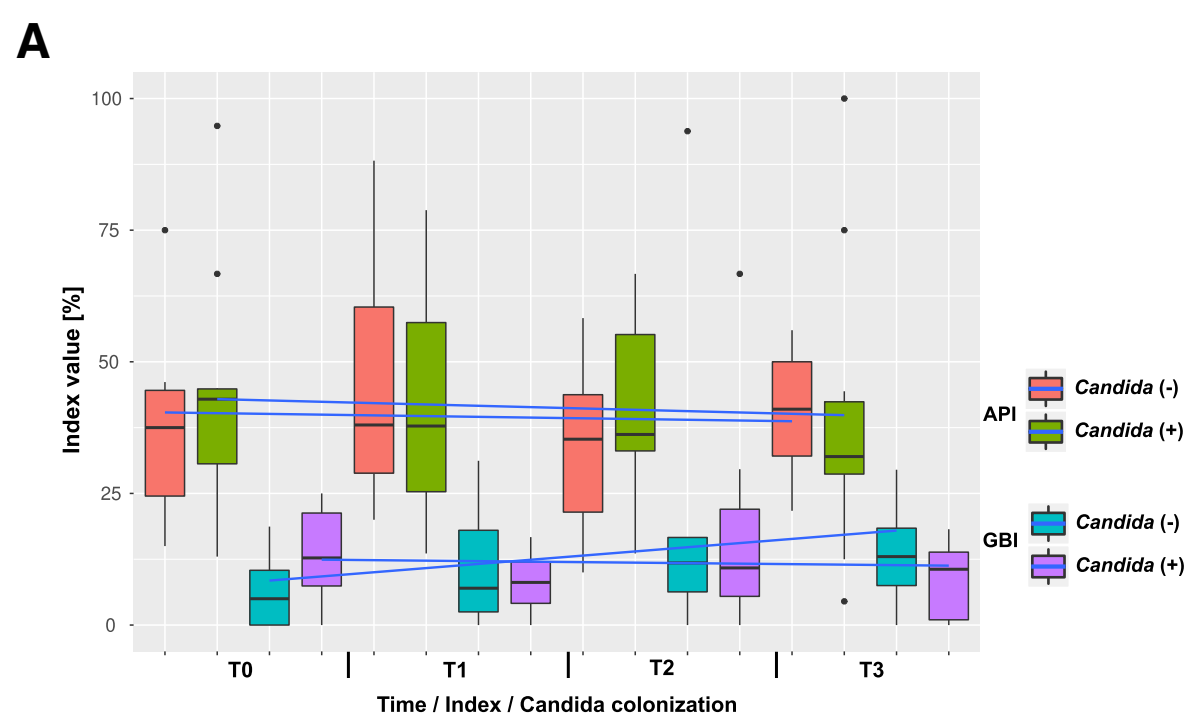

B

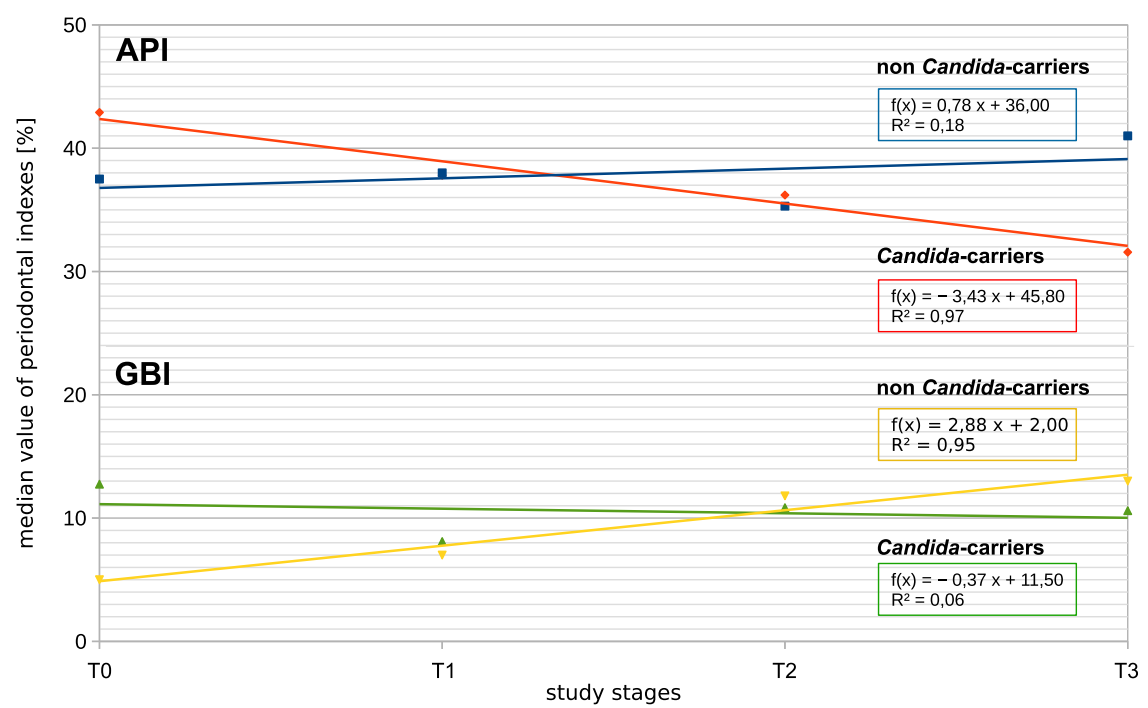

Fig. 2 a Changes in API and GBI indices on the duration of the study among Candida positive and Candida negative patients. Blue line connects the average value. $\mathbf{b}$ The same correlation as in Fig. 2, but showing the correlation with median value among Candida positive and Candida negative patients

Our findings regarding the upward trend of yeast growth during orthodontic treatment are consistent with the data presented in the literature [16-19]. The study of Zheng et al. [16] showed that the CFU/ml increases in users of fixed orthodontic appliances after 2 months of treatment. Lee et al. [19], in their long-term research (involving almost 12 months of observation), showed that the amount of Candida albicans increases after bracket bonding and peaks in the fifth month of treatment, before slightly decreasing and approaching the maximum again at the end of the first year of treatment. Arslan et al. [17], on the basis of 1 year of observation of a group of orthodontic patients, stated that a statistically significant increase in the Candida population occurs in the first month of orthodontic treatment. A decrease in the amount of Candida DNA in wash mouth samples was only found in Bergamo et al. [20] ( $N=15$, mean age $17.53 \pm 8.0$ years), who employed 90 days of observation after bracket application; this study group was heterogenous, consisting of 14 women and a single male. Unfortunately, the authors of none of these studies described patient preparation procedures, such as toothbrushing. Only Zheng et al. [16] stated that patients had had breakfast and had undergone their regular morning oral hygiene routine. Our recommendations were typical of laboratory tests: patients reported on an empty 


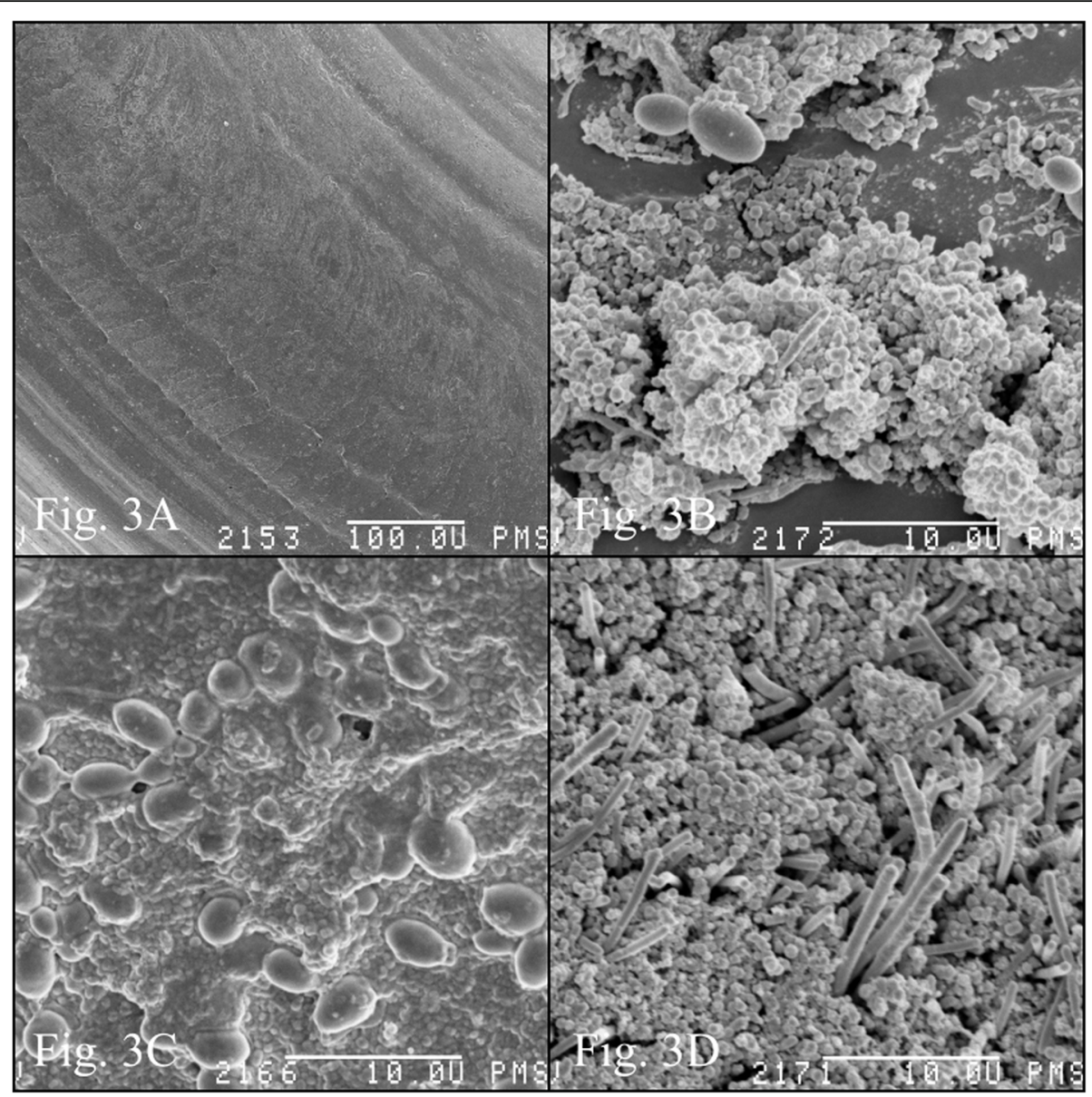

Fig. 3 a The electron microscopy image of clear orthodontic ligatures: no microbial contamination. b, c, d Orthodontic ligatures topography after four weeks' in the oral cavity. b Budding yeast cells. c Yeast cells in dense bacterial biofilm. $\mathbf{d}$ Bacterial biofilm, with rods rising straight from cocci layer

stomach, without having brushed their teeth. This is one of the methodological variable factors that may greatly affect results of the study.

The species profile obtained in our study is similar to that of Hägg and al [18]. and of Lee at al [19].: Candida albicans dominates and C. guilliermondii and C. tropicalis were isolated in small percentage. This may serve as a guideline for choosing antibiotic therapy for patients affected by oral mycosis. An interesting phenomenon, not noted by other authors, was the change in colonizing species seen in two patients over the course of the study (in K.J., C. albicans was replaced by C. tropicalis; and in D.M., strains of C. guilliermondii led to high biofilm formation; see Fig. 4). Further research may be needed to better understand the intraspecies relationships of $\mathrm{Can}$ dida yeast.

Conventional orthodontic brackets have many retention areas that impede proper oral hygiene, thus leading to greater plaque build-up. In our study, the mean number of Candida CFUs had a tendency to grow. However, the API and GBI did not show significant changes. Interestingly, statistically significant fungal growth was seen in patients whose API decreased (indicating that oral hygiene improved) during subsequent visits, whereas patients with increasing GBI (periodontal inflammation) had lower yeast carriage. This leads to the conclusion that Candida sp. proliferated less in patients with an altered periodontium i.e., these were probably more colonized by bacteria. Similarly, no differences in plaque index (PI), gingival index (GI), or gingival bleeding index (GBI) during the use of fixed orthodontic appliances were observed by Bergamo et al. [20]. However, Hägg et al. [18] found a statistically significant increase in PI during the second and third visits after beginning orthodontic treatment with brackets.

One of the factors in Candida virulence is the ability of these yeasts to form biofilm on abiotic surfaces, including components of orthodontic appliances. The formation of a biofilm protects the cells forming it against the adverse effects of the environment, including 


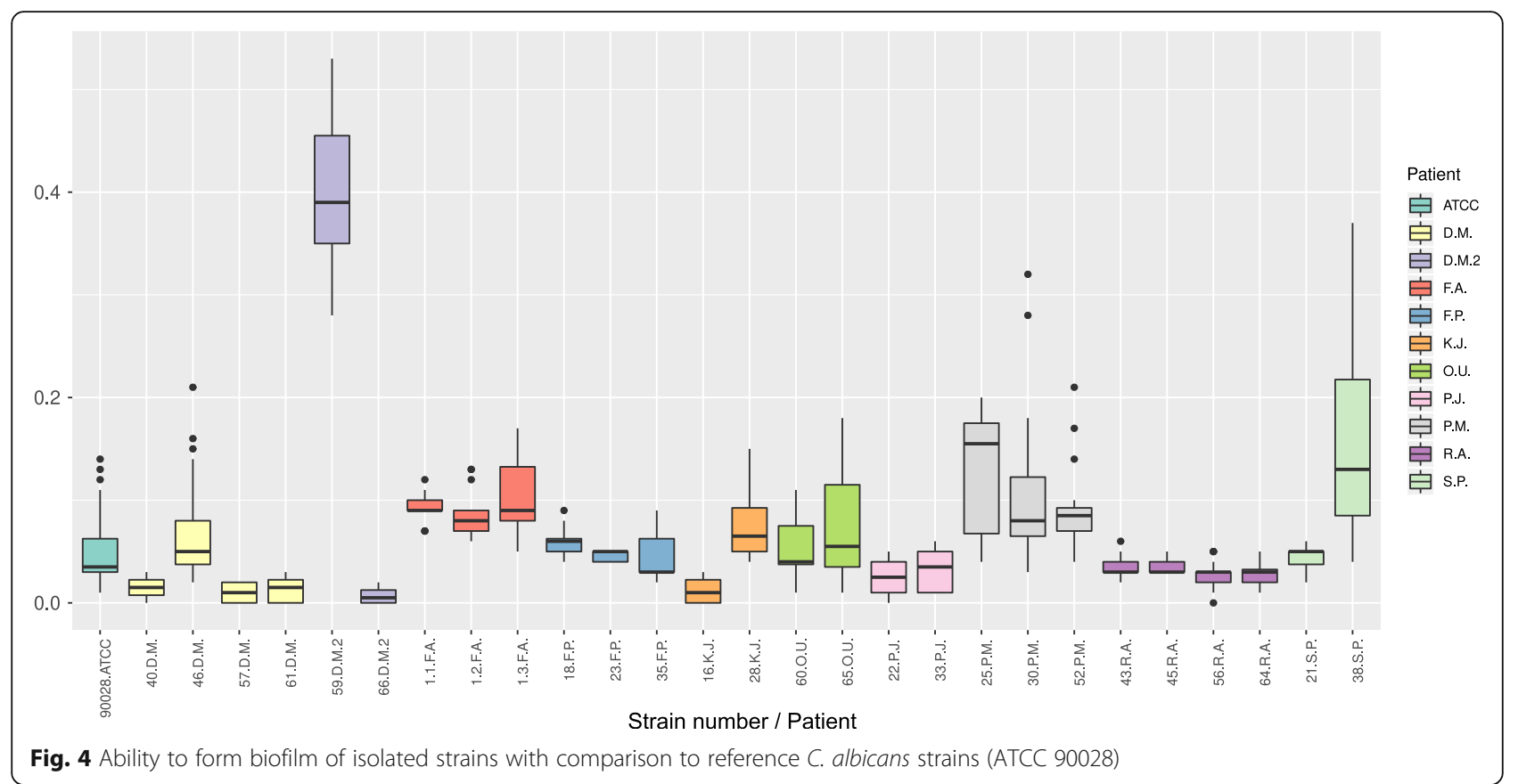

antimicrobial drugs. Few studies have taken up the topic of the multiplication of microorganisms on orthodontic elastomers [5, 21]. Casaccica et al. [4] showed that the elastomers used in orthodontics are manufactured with due care and do not pose a biological threat. SEM images of the topography of uninfected elastic ligatures presented in this publication also confirm the absence of microorganisms. The surface of elastic ligatures however enables the formation of architecturally complex and species-rich biofilms, and is rapidly colonized during orthodontic treatment.

It would seem that strains isolated from patients colonized while using orthodontic appliances will form a biofilm well. For our patients, the level of colonization before treatment was high, and during the course of treatment, the ability to create biofilm did not change, except in sporadic cases. This indicates that, while the number of yeasts including those derived from biofilms produced in the oral cavity increased, the biofilm-forming potential (virulence) of the isolated strains did not change. It can thus be concluded that, while oral appliances promote colonization by yeasts, they do not increase their virulence. Appropriate antifungal prophylaxis should thus permit control of the development of oral mycosis during long-term orthodontic therapy. However, in vivo biofilm formation may not coincide with in vitro biofilm formation capacity. The model does not take into account all factors (especially the presence of bacteria). The Candida pathogenicity (biofilm formation) described in the literature thus does not coincide with the pathogenicity of strains in vivo.

\section{Conclusions}

- Treatment with orthodontic appliances promotes colonization by Candida yeast.

- Yeast colonization is variable over time in terms of strain and species of fungi, with the dominance of Candida albicans.

- In patients who are carriers, the API value decreases over time, and in uncolonized patients the GBI value increases; this may have predictive significance for the development of oral candidiasis during orthodontic treatment, but further research is required to confirm these relationships and determine the cut-off point.

- Strains isolated from orthodontically treated patients do not show increased biofilm-forming activity.

\section{Abbreviations}

API: Approximal plaque index; GBI: Gingival bleeding index; SD: Standard deviation; CFU: Colony-forming unit; RPMI: Roswell Park Memorial Institute; MOPS: 3-morpholinopropane-1-sulfonic acid; PBS: Phosphate buffered saline; PI: Plaque index; Gl: Gingival index; ATCC: American-Type Culture Collection

\section{Acknowledgements}

This study was supported by a subsidy from the Polish Ministry of Science and Higher Education to the Jagiellonian University to maintain research potential (Program No. K/ZDS/003737).

The authors would like to thank Mrs. Katarzyna Świeży for help with the scanning electron microscope photography.

\section{Authors' contributions}

KG: qualified patients to the study, collected clinical material, analyzed and interpreted the data, designed the data, designed the tests, wrote the manuscript, reviewed the selected literature search, and critically revised of the article. PK: performed the tests, analyzed and interpreted the data, carried out the statistical analysis, wrote the manuscript, reviewed the selected literature, and critically revised the article. AHP: analyzed and 
interpreted the data and critically revised the article. JEL: analyzed and interpreted the data and critically revised the article. KTĆ: performed some of the tests, performed the scanning electron microscope photography, analyzed and interpreted the data, and critically revised the article. BWL: designed the study and critically revised the article. All authors have read and approved the final manuscript.

\section{Funding}

The funder played no role in study design, in the collection, analysis, or interpretation of the data, in the writing of the report, or in the decision to submit the article for publication.

\section{Availability of data and materials}

The datasets used and analyzed in this study are available from the corresponding author on reasonable request.

\section{Ethics approval and consent to participate}

The authors confirm that the ethical policies of the journal, as noted on the journal's author guidelines page, have been adhered to. This study was approved by the Bioethics Committee of Jagiellonian University, Kraków, Poland (No. KBET/121/B/2012, 24th March 2012), and the study took place from October 2013 to December 2014. Before beginning work, we orally explained our study objectives and procedures to all participants, and obtained their permission to use their specimens in our study. Meanwhile, a written informed consent form was signed by each participant aged 16 years and over. Written informed consent was also obtained from the parents or guardians of participants under 16 years old.

\section{Consent for publication}

Not applicable.

\section{Competing interests}

The authors declare that they have no competing interests.

\section{Author details}

'Department of Orthodontics, Dental Institute, Faculty of Medicine, Jagiellonian University Medical College, ul. Montelupich 4/108, 31-155 Kraków, Poland. ${ }^{2}$ Department of Mycology, Chair of Microbiology, Faculty of Medicine, Jagiellonian University Medical College, Kraków, Poland. ${ }^{3}$ Department of Prosthodontics, Dental Institute, Faculty of Medicine, Jagiellonian University Medical College, Kraków, Poland.

Received: 1 April 2020 Accepted: 30 September 2020 Published online: 10 October 2020

\section{References}

1. Lucchese A, Bondemark L, Marcolina M, Manuelli M. Changes in oral microbiota due to orthodontic appliances: A systematic review. J Oral Microbiol. 2018;10:1476645. https://doi.org/10.1080/20002297.2018.1476645.

2. Klaus K, Eichenauer J, Sprenger R, Ruf S. Oral microbiota carriage in patients with multibracket appliance in relation to the quality of oral hygiene. Head Face Med. 2016;12(1):1-7. https://doi.org/10.1186/s13005-016-0125-x.

3. Tooyama H, Matsumoto T, Hayashi K, Kurashina K, Kurita H, Uchida M, et al. Candida concentrations determined following concentrated oral rinse culture reflect clinical oral signs. BMC Oral Health. 2015;24(15):150. https:// doi.org/10.1186/s12903-015-0138-z.

4. Casaccia GR, Gomes JC, Alviano DS, De Oliveira Ruellas AC, Sant'Anna EF. Microbiological evaluation of elastomeric chains. Angle Orthod. 2007;77(5): 890-3.

5. Magno AFF, Enoki C, Ito IY, Matsumoto MAN, Faria G, Nelson-Filho P. In-vivo evaluation of the contamination of super slick elastomeric rings by Streptococcus mutans in orthodontic patients. Am J Orthod Dentofac Orthop. 2008;133(4 Suppl):104-9.

6. Sampaio AA, Souza SE, Ricomini-Filho AP, Del Bel Cury AA, Cavalcanti YW, Cury JA. Candida albicans increases dentine demineralization provoked by Streptococcus mutans biofilm. Caries Res. 2019;53(3):322-31.

7. Pellizzaro D, Polyzois G, MacHado AL, Giampaolo ET, Sanitá PV, Vergani CE. Effectiveness of mechanical brushing with different denture cleansing agents in reducing in vitro Candida albicans biofilm viability. Braz Dent J. 2012;23(5):547-54.
8. van Wyk C, Steenkamp V. Host factors affecting oral candidiasis. S Afr J Epidemiol Infect. 2011;26(1):18-21.

9. Scully C, Ei-Kabir M, Samaranayake LP. Candida and oral candidosis: a review. Crit Rev Oral Biol Med. 1994;5(2):125-57.

10. Bastiaan RJ, Reade PC. The prevalence of Candida albicans in the mouths of tobacco smokers with and without oral mucous membrane keratoses. Oral Surg Oral Med Oral Pathol. 1982;53(2):148-51 Available from: https://www. ncbi.nlm.nih.gov/pubmed/7036031. Cited 2020 Mar 16.

11. Rindum $J \mathrm{~L}$, Stenderup A, Holmstrup P. Identification of Candida albicans types related to healthy and pathological oral mucosa. J Oral Pathol Med. 1994;23(9):406-12 Available from: https://www.ncbi.n/m.nih.gov/pubmed/ 7823301. Cited 2020 Mar 16.

12. Hibino K, Wong RWK, Hägg U, Samaranayake LP. The effects of orthodontic appliances on candida in the human mouth. Int J Paediatr Dent. 2009;19(5): 301-8.

13. Foundation RCTR. R: A Language and Environment for Statistical Computing, vol. 2; 2013. https://www.R-project.org. Available from: https:// www.gnu.org/copyleft/gpl.html. Cited 2020 Mar 16.

14. Pohlert T. The Pairwise Multiple Comparison of Mean Ranks Package (PMCM R). R Package. 2014;27 Available from: https://cran.r-project.org/package= PMCMR. Cited 2020 Mar 16.

15. Szymańska J, Wójtowicz A, Malm A. Assessment of Candida spp. frequency in the oral cavity ontocenosis of healthy individuals in different age groups. J Pre-Clinical Clin Res. 2016;10(2):91-4.

16. Zheng Y, Li Z, He X. Influence of fixed orthodontic appliances on the change in oral candida strains among adolescents. J Dent Sci. 2016;11(1): 17-22. https://doi.org/10.1016/j.jds.2014.02.001

17. Gündüz Arslan S, Akpolat N, Kama JD, Özer T, Hamamci O. One-year followup of the effect of fixed orthodontic treatment on colonization by oral candida. J Oral Pathol Med. 2008;37(1):26-9.

18. Hägg U, Kaveewatcharanont P, Samaranayake YH, Samaranayake LP. The effect of fixed orthodontic appliances on the oral carriage of Candida species and Enterobacteriaceae. Eur J Orthod. 2004;26(6):623-9.

19. Lee W, Low BKM, Samaranayake LP, Hagg U. Genotypic variation of Candida albicans during orthodontic therapy. Front Biosci. 2008;13:3814-24. https:// doi.org/10.2741/2970.

20. Bergamo AZN, de Oliveira KMH, Matsumoto MAN, do Nascimento C, Romano FL, da Silva RAB, et al. Orthodontic appliances did not increase risk of dental caries and periodontal disease under preventive protocol. Angle Orthod. 2019;89(1):25-32.

21. Baboni FB, Guariza Filho O, Moreno AN, Rosa EAR. Influence of cigarette smoke condensate on cariogenic and candidal biofilm formation on orthodontic materials. Am J Orthod Dentofac Orthop. 2010;138(4):427-34.

\section{Publisher's Note}

Springer Nature remains neutral with regard to jurisdictional claims in published maps and institutional affiliations.

Ready to submit your research? Choose BMC and benefit from:

- fast, convenient online submission

- thorough peer review by experienced researchers in your field

- rapid publication on acceptance

- support for research data, including large and complex data types

- gold Open Access which fosters wider collaboration and increased citations

- maximum visibility for your research: over $100 \mathrm{M}$ website views per year

At $\mathrm{BMC}$, research is always in progress.

Learn more biomedcentral.com/submissions 